\title{
ON MAXIMAL $k$-IDEALS OF SEMIRINGS
}

\author{
M. K. SEN AND M. R. ADHIKARI \\ (Communicated by Maurice Auslander)
}

\begin{abstract}
For a semiring $S$ with commutative addition, conditions are considered such that $S$ has nontrivial $k$-ideals or maximal $k$-ideals, among others, by the help of the congruence class semiring $S / A$ defined by an ideal $A$ of $S$. Moreover, all maximal $k$-ideals of the semiring of nonnegative integers are described.
\end{abstract}

\section{Preliminaries}

A semiring $S$ is defined as an algebra $(S,+, \cdot)$ such that $(S,+)$ and $(S, \cdot)$ are semigroups connected by $a(b+c)=a b+a c$ and $(b+c) a=b a+c a$ for all $a, b, c \in S$. A semiring $S$ may have an identity 1 [a zero $o$ ], defined by $1 a=a 1=a[o+a=a+o=a]$ for all $a \in S$. If there is an element $O \in S$ satisfying $O a=a O=O$ for all $a \in S$, it is called multiplicatively absorbing or simply absorbing. Such an element satisfies $O+O=O$, but it need not be a zero of $S$, whereas a zero $o$ of $S$ need not even satisfy $o o=o$. Clearly, a semiring has an absorbing zero iff it has elements $O$ and $O$ which coincide.

A subset $A \neq \varnothing$ of a semiring $S$ is called an ideal of $S$ iff $a+b \in A$, $s a \in A$, and $a s \in A$ hold for all $a, b \in A$ and all $s \in S$. An ideal $A$ of $S$ is called proper iff $A \subset S$ holds, where $\subset$ denotes proper inclusion, and a proper ideal $A$ is called maximal iff there is no ideal $B$ of $S$ satisfying $A \subset B \subset S$. Obviously, a semiring $S$ contains an ideal $A$ consisting of one element iff $S$ has an absorbing element $O$, and then $A=\{O\}$ is the only ideal of this kind. Finally, an ideal $A$ of $S$ is called trivial, iff $A=S$ holds or $A=\{O\}$, the latter clearly if $S$ has an absorbing element. To deal with both cases simultaneously, we introduce the notion $S^{\prime}$ by $S^{\prime}=S \backslash\{O\}$ if $S$ has an absorbing element, and $S^{\prime}=S$ otherwise.

In this paper we only consider semirings $S$ for which $(S,+)$ is commutative. If also $(S, \cdot)$ is commutative, $S$ is called a commutative semiring. Moreover, to avoid trivial exceptions, each semiring $S$ is assumed to have at least two elements.

Using only commutativity of addition, the following concepts and statements, essentially due to $[1,2,4]$, are well known. For each ideal $A$ of a semiring $S$

Received by the editors January 22, 1991 and, in revised form, October 25, 1991.

1991 Mathematics Subject Classification. Primary 16Y60.

Key words and phrases. Semiring, $k$-ideals, maximal $k$-ideals, completely prime $k$-ideals. 
the $k$-closure $\bar{A}$ of $A$ defined by

$$
\bar{A}=\left\{\bar{a} \in S \mid \bar{a}+a_{1}=a_{2} \text { for some } a_{i} \in A\right\}
$$

is an ideal of $S$ satisfying $A \subseteq \bar{A}$ and $\overline{\bar{A}}=\bar{A}$. An ideal $A$ of $S$ is called a $k$-ideal of $S$ iff $A=\bar{A}$ holds. Clearly, $S$ is a $k$-ideal for each semiring $S$; however, if $S$ has an absorbing element $O$, the ideal $\{O\}$ need not be a $k$-ideal of $S$. There are examples for $\{O\} \subset \overline{\{O\}} \subset S$ and $\{O\} \subset \overline{\{O\}}=S$, whereas $\{O\}=\overline{\{O\}}$ holds if $O$ is an absorbing zero of $S$. A $k$-ideal $A \subset S$ is called a maximal $k$-ideal of $S$ if there is no $k$-ideal $B$ of $S$ satisfying $A \subset B \subset S$. Note that a maximal $k$-ideal of $S$ need not be a maximal ideal of $S$ (cf. Remark 4.2).

Moreover, each ideal $A$ of $S$ defines a congruence $\rho_{A}$ on $(S,+, \cdot)$ by

$$
\rho_{A}=\left\{(x, y) \in S \times S \mid x+a_{1}=y+a_{2} \text { for some } a_{i} \in A\right\} .
$$

The corresponding congruence class semiring $S / \rho_{A}$, consisting of the classes $x \rho_{A}$, is also denoted by $S / A$. The $k$-closure $\bar{A}$ of $A$ is such a congruence class, and $\bar{A}$ is the absorbing zero of $S / A$, regardless of whether $S$ has a zero $O$ or an absorbing element $O$ (which implies $o \rho_{A}=\bar{A}$ or $O \rho_{A}=\bar{A}$, respectively). Moreover, $\rho_{A}$ and $\rho_{\bar{A}}$, and hence $S / A$ and $S / \bar{A}$ coincide.

\section{MaXimal $k$-IDEALS}

Theorem 2.1. Let $S$ be a semiring such that $S=\left(a_{1}, \ldots, a_{n}\right)$ is a finitely generated ideal of $S$. Then each proper $k$-ideal $A$ of $S$ is contained in $a$ maximal $k$-ideal of $S$.

Proof. Let $\mathfrak{B}$ be the set of all $k$-ideals $B$ of $S$ satisfying $A \subseteq B \subset S$, partially ordered by inclusion. Consider a chain $\left\{B_{i} \mid i \in I\right\}$ in $\mathfrak{B}$. One easily checks that $B=\bigcup_{i \in I} B_{i}$ is a $k$-ideal of $S$, and $S=\left(a_{1}, \ldots, a_{n}\right)$ implies $B \neq S$, and hence $B \in \mathfrak{B}$. So by Zorn's lemma, $\mathfrak{B}$ has a maximal element as we were to show.

Corollary 2.2. Let $S$ be a semiring with identity 1. Then each proper $k$-ideal of $S$ is contained in a maximal $k$-ideal of $S$.

The proof is immediate by $S=(1)$.

Definition 2.3. A semiring $S$ is said to satisfy condition (C) iff for all $a \in S^{\prime}$ and all $s \in S$ there are $s_{1}, s_{2} \in S$ such that

$$
s+s_{1} a=s_{2} a
$$

holds. Clearly, if $S$ has an identity 1 , then (C) is equivalent to the following condition $\left(\mathrm{C}^{\prime}\right)$ :

$$
1+s_{1} a=s_{2} a
$$

holds for each $a \in S^{\prime}$ and suitable $s_{1}, s_{2} \in S$.

Example 2.4. Let $P$ be the set of all nonnegative rational numbers. Then $(P,+, \cdot)$ with the usual operations, as well as $\left(P^{\prime},+, \cdot\right)$, are semirings with 1 as identity satisfying condition $\left(C^{\prime}\right)$. The same is true, more generally, for each positive cone $P$ of a totally ordered skew-field (cf. [3, Chapter VI]).

Example 2.5. Let $\mathbb{N}$ be the set of all nonnegative integers. Define $a+b=$ $\max \{a, b\}$, and denote by $a \cdot b$ the usual multiplication. Then $(\mathbb{N},+, \cdot)$ is a 
semiring with 1 as identity, which satisfies $\left(\mathrm{C}^{\prime}\right)$ since $1+a=a$ holds for all $a \in S^{\prime}$.

Lemma 2.6. If a semiring $S$ with an absorbing zero $O$ satisfies condition (C), then $a b=O$ for $a, b \in S$ implies $a=O$ or $b=O$.

Proof. By way of contradiction, assume $a b=O$ and $a \neq O \neq b$. Then $s+s_{1} a=s_{2} a$, according to (C), yields $s b+s_{1} a b=s_{2} a b$, i.e., $s b=O$ for all $s \in S$. Consequently, $x+s_{3} b=s_{4} b$ implies $x=O$ for all $s_{3}, s_{4} \in S$, which contradicts (C) applied to the element $b \in S^{\prime}$.

Theorem 2.7. Let $S$ be a semiring. Then condition $(\mathrm{C})$ implies that $S$ contains only trivial $k$-ideals. The converse is true if $(S, \cdot)$ is commutative, and, provided that $S$ has an absorbing element $O, S a=\{s a \mid s \in S\} \neq\{O\}$ holds for all $a \in S^{\prime}$.

Proof. Assume that $S$ satisfies (C). Let $A$ be a $k$-ideal of $S$ which contains at least one element $a \in S^{\prime}$. Then $s+s_{1} a=s_{2} a$, according to (C), implies $s \in A$ for each $s \in S$, i.e., $A=S$. For the converse, our supplementary assumptions on $S$ yield that $S a$ is an ideal of $S$ and that $S a \neq\{O\}$ holds for each $a \in S^{\prime}$ if $S$ has an absorbing element $O$. Now assume that $S$ has only trivial $k$-ideals. Then the $k$-ideal $\overline{S a}$ coincides with $S$ for each $a \in S^{\prime}$, regardless of whether $S$ has an element $O$ or not. Now,

$$
\overline{S a}=\left\{s \in S \mid s+s_{1} a=s_{2} a \text { for some } s_{i} \in S\right\}=S
$$

states that $S$ satisfies condition (C).

Corollary 2.8. Let $S$ be a commutative semiring with identity. Then $S$ has only trivial $k$-ideals iff it satisfies condition $\left(\mathrm{C}^{\prime}\right)$.

Proof. It was already stated that $\left(\mathrm{C}^{\prime}\right)$ is equivalent to $(\mathrm{C})$ if $S$ has an identity 1 , and $a=1 a \in S a$ implies $S a \neq\{O\}$ for all $a \in S^{\prime}$ in the case that $S$ has an absorbing element $O$. Hence the corollary follows from Theorem 2.7.

Theorem 2.9. Let $S$ be a commutative semiring with identity 1 and $A$ a proper $k$-ideal of $S$. Then $A$ is maximal iff the semiring $S / A=S / \rho_{A}$ satisfies condition $\left(\mathbf{C}^{\prime}\right)$.

Proof. Suppose $A$ is a maximal $k$-ideal of $S$. Then $A$ is the absorbing zero of $S / A$ and $1 \rho_{A}$ is its identity. Consider any $c \rho_{A} \in(S / A)^{\prime}$. Then $c \notin A$ holds, and the smallest ideal $B$ of $S$ containing $c$ and $A$ consists of all elements $s c, a$, and $s c+a$ for $s \in S$ and $a \in A$. From $A \subset B$ it follows $\bar{B}=S$, and hence $1+b_{1}=b_{2}$ for suitable elements $b_{1}, b_{2} \in B$. To avoid the discussion of different cases, we add $1 c+a$ with an arbitrary element $a \in A$ to $1+b_{1}=b_{2}$ and obtain

$$
1+s_{1} c+a_{1}=s_{2} c+a_{2}, \quad \text { i.e., } 1 \rho_{A}+\left(s_{1} \rho_{A}\right)\left(c \rho_{A}\right)=\left(s_{2} \rho_{A}\right)\left(c \rho_{A}\right)
$$

for suitable $s_{i} \in S$ and $a_{i} \in A$. This shows that $S / A$ satisfies $\left(C^{\prime}\right)$.

Conversely, assume $\left(C^{\prime}\right)$ for $S / A$, and let $B$ be a $k$-ideal of $S$ satisfying $A \subset B$. Then there is an element $c \in B \backslash A$, and $c \rho_{A} \in(S / A)^{\prime}$ yields $\left(1+s_{1} c\right) \rho_{A}=\left(s_{2} c\right) \rho_{A}$ for suitable elements $s_{i} \in S$ by $\left(\mathrm{C}^{\prime}\right)$. Hence $1+s_{1} c+a_{1}=$ $\underline{s_{2}} c+a_{2}$ holds for some $a_{i} \in A$, i.e., $1+b_{1}=b_{2}$ for $b_{1}, b_{2} \in B$. This shows $\bar{B}=S$ and that $A$ is a maximal $k$-ideal of $S$. 


\section{COMPLETELY PRIME $k$-IDEALS}

Recall that an ideal $A$ of a semiring $S$ is called completely prime (cf., e.g., [5]) iff $a b \in A$ implies $a \in A$ or $b \in A$ for all $a, b \in S$.

Proposition 3.1. Let $S$ be a commutative semiring with identity. Then each maximal $k$-ideal $A$ of $S$ is completely prime.

Proof. By Theorem 2.9, the semiring $S / A$ satisfies the condition $\left(\mathrm{C}^{\prime}\right)$ and hence (C). Since $S / A$ has $A$ as its absorbing zero, we can apply Lemma 2.6 and obtain that $S / A$ has no zero-divisors. Hence $a \rho_{A} \neq A$ and $b \rho_{A} \neq A$ imply $(a b) \rho_{A} \neq A$, i.e., $a \notin A$ and $b \notin A$ imply $a b \notin A$ as we were to show.

Concerning the converse of Proposition 3.1, we show that a completely prime ideal $A$ of a commutative semiring $S$ with identity need not be a $k$-ideal, and if it is one, $A$ need not be a maximal $k$-ideal of $S$.

Example 3.2. Let $S$ be the set of all real numbers $a$ satisfying $0<a \leq 1$, and define $a+b=a \cdot b=\min \{a, b\}$ for all $a, b \in S$. Then $(S,+, \cdot)$ is easily checked to be a commutative semiring with 1 as identity. Each real number $r$ such that $0<r<1$ defines an ideal $A=\{a \in S \mid a \leq r\}$ of $S$ which is obviously completely prime. However, $r+1=r$ together with $r \in A$ and $1 \notin A$ show that $A$ is not a $k$-ideal of $S$. The same is true if one includes 0 in these considerations (in this case 0 is an absorbing element but not a zero of $S \cup\{0\}$ ), but also if one adjoins 0 as an absorbing zero to $S$ (cf., e.g., [7, Lemma 1.3]).

Example 3.3. The polynomial ring $\mathbb{Z}[x]$ over the ring $\mathbb{Z}$ of integers contains the subsemiring

$$
S=\mathbb{N}[x]=\left\{f(x)=\sum_{i=0}^{n} a_{i} x^{i} \mid a_{i} \in \mathbb{N}\right\},
$$

which is clearly commutative and has $1 \in \mathbb{N}$ as its identity. The ideal $A=(x)$ of $S$ consists of all $f(x) \in S$ such that $a_{0}=0$ holds. Obviously, $A$ is completely prime and a $k$-ideal of $S$. Now consider the set $B$ consisting of all $f(x) \in S$ for which $a_{0}$ is divisible by 2 . Clearly, $B$ is a $k$-ideal of $S$, and $A \subset B \subset S$ shows that $A$ is not a maximal $k$-ideal.

\section{Maximal $k$-ideals of $\mathbb{N}$}

In this section we consider the semiring $(\mathbb{N},+, \cdot)$ of nonnegative integers with respect to their usual operations.

Proposition 4.1. The semiring $\mathbb{N}$ has exactly the $k$-ideals $(a)=\{n a \mid n \in \mathbb{N}\}$ for each $a \in \mathbb{N}$. Consequently, the maximal $k$-ideals of $\mathbb{N}$ are given by $(p)$ for each prime number $p$.

Proof. Obviously, each ideal $(a)$ of $\mathbb{N}$ is a $k$-ideal. Now assume that $A \neq(0)$ is a $k$-ideal of $\mathbb{N}$. Let $a$ be the smallest positive integer contained in $A$, and $b$ any element of $A$. Then $b=q a+r$ holds for some $q \in \mathbb{N}^{\prime}$ and $r \in \mathbb{N}$ satisfying $0 \leq r<a$. Since $r$ belongs to the $k$-ideal $A$, it follows that $r=0$, and, hence, $A=(a)$. The last statement follows since $(a) \subseteq(b)$ holds iff $b$ divides $a$. 
Remark 4.2. None of the maximal $k$-ideals $(p)$ of $\mathbb{N}$ is a maximal ideal of $\mathbb{N}$. This follows since each ideal $A=(p)$ is properly contained in the proper ideal $B=\{b \in \mathbb{N} \mid b \geq p\}$ of $\mathbb{N}$.

\section{ACKNOWLEDGMENT}

Thanks to the learned referee for all the pains undertaken for the improvement of the paper.

\section{REFERENCES}

1. S. Bourne, The Jacobson radical of a semiring, Proc. Nat. Acad. Sci. U.S.A. 37 (1951), 163-170.

2. S. Bourne and H. Zassenhaus, On the semiradicals of a semiring, Proc. Nat. Acad. Sci. U.S.A. 44 (1958), 907-914.

3. L. Fuchs, Partially ordered algebraic systems, Pergamon Press, New York and Oxford, 1963.

4. M. Henriksen, Ideals in semirings with commutative addition, Amer. Math. Soc. Notices 5 (1958), 321.

5. K. Iséki, On ideals in semirings, Proc. Japan Acad. Ser. A Math. Sci. 34 (1958), 507-509.

6. D. R. Latorre, A note on quotient in semirings, Proc. Amer. Math. Soc. 24 (1970), 463-465.

7. H. J. Weinert, Seminearrings, seminearfields and their semigroup-theoretical background, Semigroup Forum 24 (1982), 231-254.

Department of Pure Mathematics, University of Calcutta, 35 Ballygunge Circular Road, CalcutTa 700019, INDia

Department of Mathematics, Burdwan University, Golapbag, Burdwan 713104, India 